\title{
PRESENTACIÓN DEL TEMA CONFLICTO Y CONVIVENCIA EN LA ESCUELA DE HOY
}

\author{
Felicidad Barreiro Fernández \\ Universidad de Santiago de Compostela \\ felicidad.barreiro@usc.es
}

Beatriz García Antelo

Universidad de Santiago de Compostela

beatriz.garcia.antelo@usc.es

Cuando hablamos de conflicto lo primero que debemos tener en cuenta es que no nos encontramos ante un concepto unívoco. Desde el imaginario social referirse al conflicto, en general, supone referirse a algo negativo, no deseable, a desencuentros, a disputas, a violencia, pero resulta fundamental cambiar esta concepción tradicional del conflicto.

Desde estas páginas defendemos la necesidad de entender de otra forma el conflicto:

Los conflictos son situaciones en las que dos o más personas entran en oposición o desacuerdo porque sus posiciones, intereses, necesidades, deseos o valores son incompatibles o son percibidos como incompatibles donde juegan un papel importante los sentimientos y las emociones y donde la relación entre las partes puede salir robustecida o deteriorada en función de cómo sea el proceso de resolución. (Torrego, 2000, p. 37).

Así pues, la palabra conflicto designa una situación compleja que puede enfrentar a individuos, grupos u organizaciones, pero no podemos definir lo que es el conflicto por lo que pueden llegar a ser sus resultados.

El conflicto resulta inevitable debido a que el ser humano es un ser social, que está destinado a vivir con otros seres humanos y, en principio, el conflicto no es ni bueno ni malo. De hecho puede ser muy educativo, pero depende del uso que hagamos de él.

El admitir que el ser humano es un ser social, conlleva aceptar que la convivencia, más que una obligación, es una necesidad. La convivencia no resulta fácil, sin embargo, se enseña, se aprende y se va construyendo. Educar para aprender a vivir con los demás se ha convertido, sin duda, en uno de los objetivos fundamentales de la educación y, a su vez, en uno de sus principales retos.

Respecto a la relevancia de educar para convivir destacar que la UNESCO, en el Informe de la Comisión Internacional (Delors y otros, 1996), se ha pronunciado de un modo categórico presentando los cuatro "pilares de la educación para el siglo XXI" que son: aprender a conocer, aprender a hacer, aprender a ser y aprender a vivir juntos. Se trata, por tanto, de orientar la educación hacia el logro de objetivos y de competencias, integrando el saber-conocer con el saber-ser, con el 
saber-hacer y con el saber-convivir como una estrategia necesaria para, entre otras cuestiones, saber afrontar conflictos, saber relacionarse con los demás y saber cooperar.

Así pues, el fomento de la convivencia se convierte en uno de los aspectos nucleares de la tarea educativa de este siglo, y además la escuela, que es un lugar de encuentro entre personas distintas con intereses, en muchas ocasiones, enfrentados, puede y debe transformarse en el lugar idóneo para aprender actitudes y conductas básicas en una convivencia libre, democrática, solidaria y participativa.

Esta construcción de la convivencia escolar resulta decisiva para la configuración de la convivencia social puesto que si convertimos los centros educativos en espacios de convivencia, será posible establecer las bases de una ciudadanía responsable, activa y crítica, capaz de conformar sociedades vertebradoras y cohesionadas.

Desde aquí queremos destacar la importancia de los conflictos y la necesidad de que sean utilizados positivamente en la educación, así como de enseñar a resolverlos. De hecho la resolución de los conflictos de forma positiva contribuirá al logro de una mejor convivencia, en la que prevalezca la comunicación y el diálogo, como formas de conseguir una sociedad pacífica, que sepa resolver sus discrepancias sin llegar a la violencia.

El número 27 de Innovación Educativa presenta un bloque monográfico dedicado a Conflicto y Convivencia compuesto por nueve artículos en los que se muestran distintas propuestas de análisis e intervención en la mejora de la convivencia escolar. Todos ellos presentan un denominador común, trabajar intencionalmente la convivencia en positivo buscando favorecer la convivencia en los centros y en las aulas.

Abre esta sección temática el trabajo titulado Los sistemas de apoyo entre iguales (SAI) y su contribución a la convivencia escolar, en el que José María Avilés Martínez, profesor de la Universidad de Valladolid, presenta los diversos Sistemas de Apoyo entre Iguales (SAI) que funcionan hoy en las escuelas e institutos señalando que todos ellos, de forma intencional y diferenciada, proporcionan protagonismo al alumnado en la gestión de su propia convivencia. Se analizan cada una de las modalidades de apoyo entre iguales (Acogida, Ayuda, Mediación, Tutoría y Mentoría), destacando las potencialidades que cada una de ellas posee, las habilidades que el alumnado desarrolla con su participación así como las necesidades formativas y las condiciones de implantación precisas para conseguir de ellas una efectividad manifiesta.

Los profesores de la Universidad de Córdoba Rosario Ortega Ruiz y Francisco Córdoba Alcaide, directora y miembro, respectivamente, del Laboratorio de Estudios sobre Convivencia y Prevención de la Violencia (LAECOVI) firman el artículo titulado El Modelo Construir la Convivencia para prevenir el acoso y el ciberacoso escolar en el que exponen el origen, la evolución y la estructura del Modelo Construir la Convivencia (MCC), un modelo teórico propuesto para intervenir en la mejora de la convivencia escolar que ha sido evaluado y del que se conocen sus resultados positivos. Precisamente en este artículo se presenta un ejemplo de la puesta en marcha del MCC en un centro de Educación Infantil y Primaria ubicado en la provincia de Córdoba que ha sido reconocido como centro promotor de convivencia positiva. 
A continuación las profesoras de la Universidad de Valencia Ana Ma Moral Mora, Amparo Pérez Carbonell, Inmaculada Chiva Sanchis y Genoveva Ramos Santana, destacan, en su trabajo $L a$ evaluación de los planes de convivencia para la reflexión de la práctica educativa, la relevancia de plantearse el uso de un proceso de reflexión en torno a la convivencia en los centros en el que se incluya a toda la comunidad escolar y proponen la evaluación como el motor del cambio que posibilite una convivencia positiva en los centros educativos. Concretamente defienden la necesidad de evaluar los planes de convivencia en los centros escolares para poder conocer, de forma holística y concreta, la situación de convivencia real. Así, de este modo, la evaluación se convierte en el proceso que puede facilitar y obtener las evidencias que permitan realizar el cambio que se necesita.

En el artículo $O$ rol do equipo de orientación específico nos trastornos da conduta José Enrique Abalo Quintela, miembro del Equipo de Orientación Específico de Pontevedra, analiza un recurso externo de apoyo a los centros educativos gallegos para gestionar adecuadamente los problemas de conducta y la convivencia: los equipos de orientación específicos (EOE). En este trabajo se indican las funciones previstas para este servicio, las líneas de intervención, así como los protocolos generales a seguir profundizando, además, en los principios en los que inciden las intervenciones.

La siguiente contribución, Aportaciones de los grupos interactivos desde la perspectiva de estudiantes y voluntariado, de Edurne Chocarro de Luis, profesora de la Universidad de La Rioja, y Sara Mollà Peña, profesora del CEIP Caballero de la Rosa, tiene como objetivo conocer las percepciones de estudiantes y el voluntariado sobre su participación en grupos interactivos, entendiendo estos como una de las actuaciones de éxito propias de Comunidades de Aprendizaje. Las autoras nos presentan la organización y el funcionamiento de los grupos interactivos en un Centro Educativo de Infantil y Primaria situado en Logroño e identifican el impacto que genera el trabajo en grupos interactivos tanto en el proceso de aprendizaje como en la convivencia.

Por su parte, los profesores de la Universidad de Lleida Pilar Rueda Carcelén, Enric Cabello Cuenca, Gemma Filella Guiu y Agnès Ros Morente, en el trabajo titulado Los programas de educación emocional HAPPY 8-12 y HAPPY 12-16 para la resolución asertiva de conflictos en niños $y$ adolescentes dan a conocer, de manera detallada, dos programas de educación emocional diseñados para entrenar las competencias emocionales en situaciones conflictivas entre iguales partiendo de la premisa de que resolver situaciones conflictivas de manera asertiva requiere del uso de la regulación emocional. Estos programas se han implementado, en las provincias de Lleida y Huesca, y evaluado en Educación Primaria (Happy 8-12) y en Educación Secundaria Obligatoria (Happy 12-16) con resultados satisfactorios, especificándose en este artículo los principales resultados cualitativos.

El siguiente trabajo, firmado por la investigadora Aixa Permuy Martínez de la Universidad de Santiago y la orientadora Begoña Cortés Ferro, también se centra en la temática de la Educación Emocional y lleva por título A coeducación das emocións como estratexia chave para a promoción da convivencia positiva: a experiencia "EmocionadXs” do IES “Xulián Magariños" (Negreira). En él se presenta el proyecto "EmocionadXs", un proyecto de educación-coeducación de las emociones para alumnado de Educación Secundaria Obrigatoria, desarrollado durante tres cursos académicos en un Instituto ubicado en la provincia de A Coruña. En el artículo se exponen algunos de los 
resultados de ese proyecto, con la intención de evidenciar los efectos positivos de la educación de las emociones en las relaciones de convivencia en los centros educativos.

A continuación el artículo de las profesoras de la Universidad de Barcelona Trinidad Donoso Vázquez, María José Rubio Hurtado y Ruth Vilà Baños, bajo el título Los espectadores y espectadoras de la ciberviolencia de género, aborda el tema del ciberacoso o ciberviolencia, más concretamente la ciberviolencia de género. Las autoras presentan los resultados de una investigación, llevada a cabo en seis comunidades autónomas, centrándose fundamentalmente en el análisis del papel que tienen las y los jóvenes cuando son observadores de ciberviolencias de género, identificando qué características de ellos/as pueden aumentar la probabilidad de actuar de un modo cómplice con el ciberagresor, y cuáles de actuar ayudando a la víctima.

El trabajo que cierra esta sección temática también profundiza en la ciberviolencia de género. En Observar la violencia 2.0: comportamiento cómplice, de la adolescencia gallega, en función del género, M José Méndez Lois, Milena Villar Varela y Felicidad Barreiro Fernández, profesoras de la Universidad de Santiago de Compostela, nos presentan los resultados de un estudio desarrollado en Galicia en el que se pretende conocer las experiencias de violencia 2.0 observadas por los y las adolescentes en entornos virtuales, analizar las posibles diferencias del comportamiento de la persona observadora en función del género e identificar factores asociados con el comportamiento de observador/a de violencias 2.0 en el colectivo adolescente.

\section{REFERENCIAS BIBLIOGRÁFICAS}

Delors, J. y otros (1996). La educación encierra un tesoro. Informe a la UNESCO de la Comisión Internacional sobre la educación para el siglo XXI. Madrid: Santillana/Ediciones UNESCO.

Torrego, J. C. (2000). Mediación de conflictos en instituciones educativas: manual para la formación de mediadores. Madrid: Narcea. 